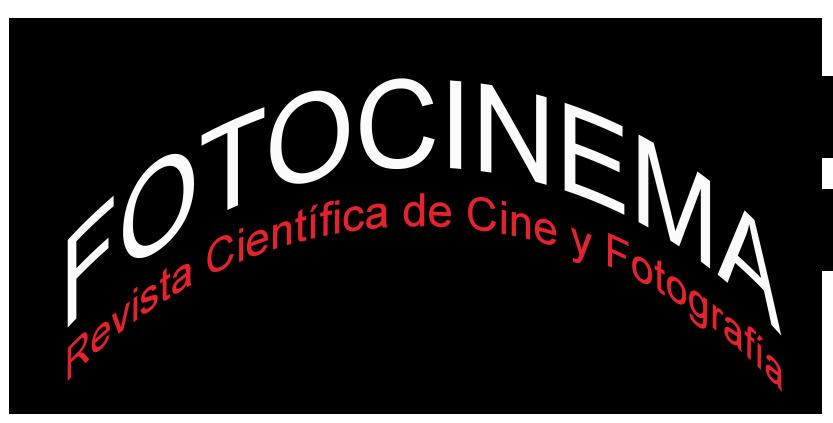

Recibido 27-10-2015 / Aceptado 02-12-2015 Publicado 29-01-2016

\title{
LA TEMPORALIDAD LONGITUDINAL EN EL CINE: DE LAS UP SERIES A BOYHOOD
}

\section{LONGITUDINAL TEMPORALITY IN CINEMA: FROM THE UP SERIES TO BOYHOOD}

\author{
Efrén Cuevas Álvarez \\ Universidad de Navarra, España \\ ecuevas@unav.es
}

\section{Resumen:}

El presente artículo analiza la "temporalidad longitudinal" en el cine. Dicha temporalidad tiene una presencia significativa en el documental así llamado, "longitudinal", que se lleva realizando desde los años sesenta hasta la actualidad. También se puede observar en las filmografías de corte autobiográfico, como las de Alan Berliner o Ross McElwee. Y recientemente el cine de ficción la ha trabajado de modo muy explícito en la exitosa Boyhood. Aquí se analizarán las pautas narrativas y temáticas que caracterizan este tipo de temporalidad, explorando los parecidos y diferencias entre las propuestas más clásicas de noficción y la reciente propuesta que plantea Boyhood desde el cine de ficción.

\begin{abstract}
:
This article analyzes the "longitudinal temporality" in cinema. This temporality has a significant presence in non-fiction cinema, in the so called "longitudinal documentary," which has being made since the Sixties up to now. It can also be found in autobiographical filmographies such as the ones of Alan Berliner and Ross McElwee. And recently, fiction cinema has explored it in a very explicit way in the successful film Boyhood. Here we will analyze the narrative and thematic features that characterize this type of temporality, exploring the likeness and differences between the more classical proposals of non-fiction cinema and the recent one posed by Boyhood from the side of fiction cinema.
\end{abstract}

Palabras clave:

Temporalidad; documental; longitudinal; autobiografía; Boyhood

Keywords:

Temporality; Documentary; Longitudinal; Autobiography; Boyhood 


\section{Introducción}

El estreno en 2014 de la película Boyhood vino acompañado de un amplio reconocimiento crítico, que colocó este film en ese pódium de "películas del año” que la opinión cinéfila construye cada año de un modo más o menos explícito. Boyhood llamó la atención por su atinado retrato de la sociedad estadounidense, por su conseguido relato generacional y por su inteligente uso del tiempo y de la elipsis. Para construir esta historia, su director Richard Linklater planteó un rodaje inusual que se prolongó durante doce años trabajando siempre con los mismos actores. Este hecho, muy comentado en la promoción y en la recepción crítica del filme, tiene como consecuencia evidente que los espectadores podemos seguir el despliegue dramático de la acción al tiempo que somos testigos del crecimiento físico y emocional de esos actores/personajes. El recurso a un tiempo tan dilatado de rodaje con los mismos actores hace que su condición de personajes se funda en cierto modo con su condición de personas reales que cambian con los años y no debido al trabajo de maquillaje y vestuario. Este enfoque resulta novedoso en el cine de ficción, como muchos han destacado, pero su estrategia temporal presenta claros ecos con propuestas más conocidas en el cine de no-ficción, de un modo muy claro con lo que se ha denominado "documental longitudinal" y de un modo más amplio con las filmografías de documentalistas que trabajan habitualmente en clave autobiográfica. ${ }^{1} \mathrm{El}$ presente artículo pretende analizar estas diferentes propuestas en lo que tienen de común y de diferente, englobándolas bajo un paraguas conceptual común que denominaremos "temporalidad longitudinal”.

\section{La temporalidad longitudinal y su aplicación al cine documental}

Adjetivar como "longitudinal" a un determinado tipo de temporalidad narrativa puede resultar un tanto ambiguo, pues lo habitual de un relato es que se despliegue de modo "longitudinal", en el sentido de lineal o

\footnotetext{
${ }^{1}$ El propio director Richard Linklater señala el parentesco de Boyhood con el documental longitudinal en una entrevista en Indiewire realizada el 15 de julio de 2014.
} 
cronológico (a excepción de los relatos planteados en entornos multimedia, también denominados relatos "no lineales", pues contienen hipertextos que nos permiten desviarnos y tomar caminos alternativos). Sin embargo, en inglés el término "longitudinal" contiene otro significado que remite directamente a lo aquí planteado, como indica el diccionario Merriam and Webster: "done by observing or examining a group of people or things over time to study how one or two particular things about them change". Este significado no está recogido en el diccionario de la Real Academia, pero tampoco contradice el sentido intuitivo de la palabra española "longitudinal", por lo que aquí hemos optado por su traducción literal. Nos encontramos ante un tipo de relato audiovisual basado en el seguimiento de una serie de personas/personajes a lo largo del tiempo. Lo esencial, por tanto, reside en la contemporaneidad entre el despliegue biográfico de esas personas y su seguimiento audiovisual.

Aplicado de modo específico a la no-ficción, no entrarían en esta categoría las biografías en las que desde el presente se recupera el pasado de un personaje recurriendo a testimonios y material de archivo. Tampoco encajarían los documentales observacionales, en tanto que se ciñen a periodos específicos, pero sin pretensión de continuar su observación a lo largo de los años. Hablaremos, por tanto, de "documental longitudinal" cuando estemos ante trabajos con carácter de serie, con varias entregas (en formato largometraje) a lo largo de los años, estrenadas en cine o televisión, en las que se aborda el seguimiento de un número limitado de personas a lo largo de un tiempo prolongado. La serie más conocida de este tipo es la bautizada como $U p$ Series, que arrancó con Seven Up, estrenada en 1964 en Gran Bretaña, en la que se seguía a catorce niños de siete años, y que ha tenido continuaciones, dirigidas por Michael Apted, cada siete años hasta la actualidad, con el estreno en 2012 de 56 Up. Pero no es el único caso, pues también se pueden encontrar series similares en otros países europeos. En Suecia destaca The Children of Jordbrö, una serie dirigida por Rainer Hartleb que produjo un filme anual entre 1972 y 1980, para luego ofrecer nuevas entregas más distanciadas hasta 1994, con un último episodio en 2006. En Alemania se 
pueden destacar varios casos: Berlin-Ecke Bundesplatz, dirigida por HansGeorg Ullrich y Detlef Gumm, que abarca de 1985 a 2004 y se centra en Berlín oeste; la serie sobre Wittstock realizada por Volker Koepp de 1975 a 1997; o The Children of Golzow, dirigida por Winfried Junge, que sigue a residentes de esta ciudad (situada en Alemania del Este hasta la caída del muro) desde 1961 a 2007, con entregas no periódicas durante esos años. En Rusia Serguéi Miroshnichenko planteó una serie siguiendo el modelo británico y apadrinada de hecho por Apted: Born in the USSR, que ha dado lugar a cuatro episodios desde 1991, estrenados también cada siete años. En España no se han dado documentales longitudinales de tan larga duración, aunque cabría mencionar a Joaquím Jordá con Numax presenta... (1980) y Veinte años después (2004), que plantea una serialidad reducida a dos entregas. ${ }^{2}$

Estos documentales longitudinales han tenido diferentes orígenes, pues no siempre comenzaron con un propósito explícito de continuar en el tiempo. Pero todos ellos comparten una serie de rasgos comunes, que aquí analizaremos brevemente siguiendo en buena medida lo planteado por Richard Kilborn (2010) en su estudio monográfico sobre el tema. Lo habitual es que arranquen con un estudio de niños o jóvenes y con una pretensión de análisis social, más en clave de grupo o clase que de individuos. Su evolución posterior les suele llevar a ir centrando su atención en los desarrollos biográficos individuales, con una perspectiva retrospectiva cada vez más marcada. Esta retrospección se va apoyando, según avanza la serie, en metraje de episodios anteriores a la hora de plantear las nuevas entregas, en lo que Kilborn (2010) denomina "the temporal shuttle" (p. 26) como rasgo propio de su estructura temporal. De esta manera el despliegue narrativo de esas vidas va tomando el control del relato, colocando en primer plano una comprensión narrativa de sus identidades personales frente al retrato social que, sin desaparecer, pasa a un segundo plano.

${ }^{2}$ Los títulos no españoles aquí mencionados provienen del estudio monográfico de Richard Kilborn sobre el tema, Taking the Long View. A Study of Longitudinal Documentary (2010), excepto en el caso ruso, sugerido por Carlos Muguiro. El título en inglés de la serie rusa es título original. De los títulos mencionados solo he tenido acceso directo a la serie británica y a las películas españolas. 
A pesar de la distinción clara entre el director y los protagonistas de la serie, la creciente cercanía que se establece entre el cineasta y esos protagonistas a lo largo de los años termina acercando estas películas a lo que Philipe Lejeune (1994) ha denominado en el ámbito escrito “autobiografía en colaboración”, que él define como la autobiografía de aquellos que no escriben (pp. 313-326), que en nuestro caso se puede traducir como la autobiografía de aquellos que no filman. De un modo cercano a los casos que Lejeune cita en literatura, en estos documentales longitudinales el creciente peso del despliegue biográfico termina por colocar al director en una posición tan cercana a sus protagonistas que se puede entender hasta cierto punto como un trabajo al servicio de un ejercicio identitario con resonancias cada vez más autobiográficas. En lo que claramente coinciden con los casos analizados por Lejeune es en el carácter ordinario de los protagonistas, lo cual remite también al creciente interés de la sociología y de la historiografía por enfoques que propongan una "historia desde abajo". Estos acercamientos plantean una sociología/historia de lo cotidiano que da acceso al pasado histórico desde enfoques alternativos a la clásica historia pública de los grandes nombres. ${ }^{3}$ Se postula de modo abierto un cambio de escala, que se centra en una pequeña comunidad, una familia o un individuo, como modo de buscar nuevos caminos para comprender mejor sociedades y tiempos pasados, un acercamiento que resulta también patente en los diversos documentales longitudinales aquí mencionados.

\section{El documental autobiográfico como relato longitudinal}

La deriva autobiográfica que ha tomado una parte significativa de la creación documental contemporánea ha adquirido en ocasiones un planteamiento similar al desplegado por los documentales longitudinales. Así se observa en algunos documentales singulares, cuya estructura interna está articulada en

\footnotetext{
3 Para un análisis de estas tendencias, véase, entre otros, Ben Highmore (ed.), Everyday Life Reader (2002), Alf Lüdtke (ed.), History of Everyday Life: Reconstructing Historical Experiences and Ways of Life (1995) y Giovanni Levi, "Sobre microhistoria" (1993, pp. 97119).
} 
torno a secuencias temporales de carácter episódico. Un caso paradigmático podría ser la película Anпа 6-18 (Анна: от 6 до 18, 1994), en la que el conocido director ruso Nikita Mikhalkov articula su retrato del país -a medio camino entre lo personal y lo colectivo- a partir de entrevistas que cada año hace a su hija Anna, desde que tiene seis años hasta que cumple dieciocho. Se observa una interesante coincidencia entre ese periodo biográfico de Anna y el del protagonista de Boyhood, si bien las estrategias y el enfoque de este filme no buscan plantear una hibridación con la ficción, sino entroncar el retrato colectivo de su país en el desarrollo temporal de su biografía familiar y personal. Otro caso notable sería el de Following Sean (2005), en el que Ralph Arlyck ofrece un retrato de la sociedad estadounidense a partir del periplo personal de Sean en su infancia y luego en su madurez, en paralelo al propio itinerario autobiográfico del director.

Pero los casos que remiten de un modo más directo a los documentales longitudinales estudiados por Kilborn son los de filmografías configuradas en su totalidad o en su mayoría por trabajos autobiográficos. No son muchos los casos en los que se da esta circunstancia, pues en bastantes ocasiones encontramos obras autobiográficas singulares en el contexto de una filmografía documental no autobiográfica o incluso en cineastas que trabajan habitualmente en el ámbito de la ficción. Un primer nombre que podría encajar aquí es el de Jonas Mekas, por el carácter diarístico de su filmografía. Sin embargo, su caso no se ajusta del todo a esta categoría, pues el carácter no retrospectivo del diario termina marcando el tono principal de sus películas, a pesar de que hay un trabajo de post-producción -textos escritos, músicas, voces en off- que sitúa sus obras a medio camino entre el diario escrito y la autobiografía canónica.4 Más interesantes resultan los casos de cineastas como Alan Berliner o Ross McElwee, dos de los cineastas más conocidos que han construido su filmografía documental sobre bases habitualmente autobiográficas. 5 Desde Intimate Stranger (1991) hasta First

\footnotetext{
$4 \mathrm{He}$ abordado con más detalle este carácter híbrido del cine de Mekas en la parte introductoria (pp. 55-57) de mi artículo "The Immigrant Experience in Jonas Mekas's Diary Films: A Chronotopic Análisis of Lost, Lost, Lost" (2006).

5 Para un estudio más amplio de estos cineastas, véase Efrén Cuevas y Carlos Muguiro (eds.),
} 
Cousin Once Removed (2012), la filmografía de Berliner está planteada como episodios de su vida -en diálogo con diferentes temas, como la relación paterno-filial, el nombre propio o el insomnio-, pero siempre entreverados con su propia biografía, con su relación con sus padres y hermana, su matrimonio, la propia paternidad, etc. Algo similar ocurre con su compatriota Ross McElwee, comenzando con Backyard (1984) hasta su último trabajo Photographic Memory (2011), con la relativa excepción de Something to Do with the Wall (1991). McElwee también hace descansar el peso de sus historias en temas diversos - como la cultura sureña del tabaco o la realidad vista a través de los medios-, pero siempre imbricadas en su propio acontecer autobiográfico.

Tanto en Berliner como en McElwee vuelve a destacar la importancia de una comprensión narrativa de la identidad, en la medida en que colocan de modo explícito el relato de sus propias vidas como generador de sentido identitario, elemento muy presente en los relatos autobiográficos, como bien señala Paul John Eakin (2008): "narrative is not merely about self, but is rather in some profound way a constituent part of self" (p. 2). Eakin explica cómo la autobiografía es de hecho una manifestación de la práctica más general de auto-narración, usada por los seres humanos para dar sentido del yo en su despliegue temporal, lo que configura un "yo extendido", según la tipología de Ulric Neisser que el propio Eakin utiliza, un yo que se proyecta hacia el pasado y el futuro, que se despliega en el tiempo y que en consecuencia se expresa en términos narrativos (Eakin, 2008, pp. 3-8). Esta comprensión narrativa de la identidad está muy vinculada a la temporalidad longitudinal que aquí estamos analizando, tanto en los documentales no autobiográficos como en los autobiográficos, pues la propia serialidad desplegada a lo largo de los años por estos trabajos introduce una dimensión reflexiva en las personas retratadas, que les enfrenta con una comprensión retrospectiva de sus vidas, que se entiende y despliega en términos narrativos. El espectador que realiza un seguimiento de estas series documentales es muy consciente García (eds.), Paisajes del yo. El cine de Ross McElwee (2008). 
de esta dimensión característica de la temporalidad longitudinal, que en buena medida constituye el principal reclamo para ese seguimiento serial.

Las estrategias temporales de estas filmografías autobiográficas coinciden grosso modo con los documentales longitudinales no autobiográficos, en lo referido principalmente al uso de la elipsis y, en menor medida, a los viajes temporales (the temporal shuttle antes mencionado). La elipsis narrativa ese salto en el tiempo del relato respecto al tiempo de la historia, en terminología genettiana (1989, p. 161)- adquiere en estas obras una importancia singular, en especial aquellas elipsis que se producen entre los diferentes episodios, que marcan el paso de los años de un modo primero y radical en el rastro que ese periodo ha dejado en la apariencia física de sus protagonistas. Esto requiere un espectador fiel a su carácter serial, capaz de plantear los puentes necesarios entre los diferentes periodos reflejados en cada película. En el caso de las series documentales antes mencionadas, ese trabajo es apoyado con frecuencia por vueltas al pasado internas, a episodios anteriores de la serie. No ocurre así en los documentales autobiográficos de Berliner o McElwee, en donde apenas se hacen explícitas esas elipsis, más allá de alguna breve mención en los comentarios en off, dejando al espectador el trabajo de construir el relato biográfico a partir de cada nueva película. En sus películas también son menos protagonistas los cambios temporales entre pasado y presente, y cuando se dan, con frecuencia no responden a un reciclaje de materiales ya mostrados en entregas anteriores, sino al empleo de material de archivo familiar o personal. Ese archivo familiar se distancia en cierto modo del uso habitual del archivo público y se acerca más a ese tiempo longitudinal de su cine autobiográfico, pues se sitúa en el despliegue temporal de sus vidas registrado por el cine. Lo que antes fue cine doméstico, y por lo tanto, restringido al ámbito de exhibición familiar, pasa ahora a formar parte de un registro público, pero insertado en una práctica autobiográfica que preserva en cierta medida su carácter íntimo o familiar. Así considerado, sí encontramos viajes temporales puntuales pero significativos en ambas filmografías, de un modo más autoconsciente si cabe en el cine de Ross McElwee. Así ocurre, por ejemplo, cuando reutiliza en 
Time Indefinite (1993) metraje doméstico de su bautizo seguido de una divertida escena de infancia con su "primer beso", filmados ambos por un tío suyo. En todo caso, estos saltos temporales no buscan de modo directo enmarcar cada nueva película en un discurso serial, como sí ocurre, por ejemplo, en las Up series dirigidas por Michel Apted.

\section{Boyhood}

Una vez analizados los rasgos configuradores de esa temporalidad longitudinal en el cine documental, no es difícil percatarse de los ecos evidentes que se perciben en el armazón cinematográfico de Boyhood, cuyo resultado final plantea una fecunda hibridación entre ficción y documental. Como ha sido ampliamente publicitado, la película se rodó durante doce años, con breves periodos de rodaje cada año. Los actores se eligieron al inicio de ese periodo temporal con el pacto de que se mantuvieran en el rodaje durante todo ese periodo (en el caso de los menores con el compromiso paterno, algo más asequible para el personaje de Samantha, la hermana del protagonista, pues está interpretada por Lorelei Linklater, a la sazón hija del director). Richard Linklater volvía cada año a encontrarse con esos actores, que por un breve tiempo interpretaban unos personajes que iban desplegando su mundo diegético a la par que los propios actores crecían y maduraban. Se produce aquí una primera hibridación entre ficción y documental que resulta fundamental para entender por qué Boyhood es diferente y cómo es experimentada por los espectadores. Muchas otras películas cuentan historias que cubren amplios periodos de tiempo en las biografías de sus protagonistas. Pero o bien son interpretados por el mismo actor, caracterizado a través del maquillaje con diferentes edades, o en casos de periodos muy amplios se recurre a actores de diferentes edades que guarden cierto parecido. Encontrarse aquí con unos actores que cambian de modo paralelo al despliegue de la diégesis durante doce años de su vida supone una experiencia espectatorial diferente, más cuando los cambios más 
sustanciales afectan al paso de la infancia a la madurez de Mason, el protagonista principal.

Sin duda resuena en este proyecto de Richard Linklater los postulados de dos clásicos de la teoría realista, André Bazin y Sigfried Kracauer, pues su apuesta creativa rezuma del ideal de cine realista que ambos autores defendían. En el caso de Kracauer (1989), se perciben ecos claros de su empeño por subrayar la capacidad del cine para rescatar la realidad física (p. 368) y por defender un cine caracterizado por su carácter de narración abierta, conectada con el flujo de la vida, con una permeabilidad que permite que la vida material fluya a través de la película (pp. 316-319). Algo que más allá de la ambientación, tan pegada a la cotidianeidad de la sociedad texana, se refleja en ese seguimiento de la evolución física de los actores convertidos en personajes. Las tesis bazinianas encuentran también un eco evidente en su idea fundacional de la capacidad del cine para momificar el cambio, liberar el tiempo de su corrupción (Bazin, 1990, p. 29), al tiempo que en su defensa de un cine que capte la ambigüedad de lo real (Bazin, 1990, pp. 94-96). La cerrada defensa del neorrealismo abanderada por Bazin encuentra también claros ecos en Boyhood, en relación con aspectos como la amalgama de actores profesionales y no profesionales, el recurso al rodaje en localizaciones reales y, sobre todo, a la primacía de la representación de la realidad sobre las estructuras dramáticas (Bazin, 1990, pp. 375-379), que otorga a los hechos "una cronología accidental y como anecdótica” (Bazin, 1990, p. 330).

El singular proceso creativo desplegado a lo largo de los doce años de rodaje introduce una segunda hibridación entre ficción y documental, vinculada más directamente con la construcción de la historia. ${ }^{6}$ Richard Linklater parte de unas líneas generales de la historia, con una premisa clara relativa al marco temporal, que abarcaría desde la infancia hasta la llegada a la universidad. Pero trabaja las líneas dramáticas aprovechando los rasgos biográficos y temperamentales de sus protagonistas, a quienes ve crecer y

${ }^{6}$ Un buen resumen de las circunstancias relacionadas con el proceso de construcción de la historia y del rodaje se puede encontrar en la síntesis, completada con numerosos enlaces, realizada por Forrest Wickman para Slate Magazine en julio de 2014. 
madurar durante el rodaje, especialmente a los dos hermanos protagonistas. Así por ejemplo el personaje de Mason incorpora aspectos biográficos del actor Ellar Coltrane, como su gusto por la fotografía o de modo más anecdótico su afición a la saga de la Guerra de las Galaxias o su recelo hacia Facebook. Linklater también introduce elementos biográficos de su propia vida y de la vida de los actores adultos: desde las canciones que toca el padre de Mason, que él mismo había compuesto e interpretado, hasta la circunstancia de que varios de ellos crecieron en hogares con padres divorciados. También cabe destacar el hecho de situar la historia en Texas, tanto en Houston como en pequeñas ciudades o entornos rurales menos conocidos, ambientes familiares para el director pues se trata de su tierra natal. Este amalgamiento de ficción y realidad pudo ir fraguándose gracias al método de escritura del guion, que partía de una plantilla muy flexible y que iba tomando forma de año en año a partir de lo rodado el año anterior y de las aportaciones autobiográficas del director y de los actores. Curiosamente Linklater ha explicado que esto no implicaba improvisación en el rodaje. El guion se trabajaba en ocasiones hasta los mismos días de los ensayos, pero una vez escrito se seguía en el rodaje sin espacio a las improvisaciones.

Otro aspecto ya apuntado que contribuye a la hibridación es el relativo a la imbricación cercana entre la vida cotidiana de la sociedad estadounidense de esos años y la historia de ficción desplegada en Boyhood. Observamos al padre de Mason discutir sobre la guerra de Irak o pedir ayuda a sus hijos en la campaña a favor de Obama, sucesos históricos que se están produciendo al tiempo que la propia vida de los actores y que el propio rodaje de la película. En ocasiones Linklater también incrusta el rodaje en acontecimientos reales, como es el caso de la publicación de una nueva entrega de Harry Potter o el partido de beisbol de los Astros de Houston.

Estos mimbres creativos configuran una película que presenta una temporalidad "longitudinal" muy similar a la observada en los documentales longitudinales y en las filmografías autobiográficas antes analizadas. Si bien en este caso no nos encontramos con una serie, sino con una película singular, su estructura temporal presenta, no obstante, una clara estructura 
episódica, en la que se va desplegando una leve progresión dramática, pero sin que llegue a predominar sobre su estructura temporal longitudinal, en la que cada episodio reclama un protagonismo similar. En este sentido cabe también afirmar que domina en Boyhood una mirada de corte observacional -por utilizar terminología afín a la creación documental-, atenta al despliegue de la vida cotidiana, aunque sin excluir puntos de giro y conflictos dramáticos que van haciendo madurar y progresar a los diferentes personajes.

Para construir este armazón narrativo, Linklater maneja con maestría una figura narrativa fundamental en este relato: la elipsis temporal. La película salta literalmente de año en año, de secuencia en secuencia, sin apenas indicar esas elipsis, sin recurrir a fundidos a negro que las señalicen o a diálogos que sitúen cómodamente al espectador tras cada cambio temporal. Se trata de una estrategia deliberadamente buscada por Linklater, que de hecho llegó a eliminar alguna transición del montaje final por resultarle demasiado obvia.7 El cineasta buscaba un relato fluido y su trabajo con las elipsis se convierte en la herramienta narrativa fundamental para conseguirlo, logrando captar en 159 minutos el retrato de una época a través de tres generaciones. Las partes omitidas del relato no resultan indiferentes, más bien adquieren una presencia propia, de un modo implícito pero concreto, gracias a la fluidez que imprime Linklater al ritmo narrativo, a ese uso de la elipsis que hace que los diferentes saltos temporales queden incorporados de un modo tan natural en la dinámica del despliegue biográfico de los personajes. Quizá lo más sorprendente es la maestría con la que Linklater combina los cambios de ritmo, las diferentes velocidades narrativas (recuperando de nuevo la terminología genettiana [1989, pp. 152171]). No existen aquí sumarios, tampoco anacronías temporales, pues la película sigue un orden cronológico lineal. Abundan los momentos tranquilos, en los que casi nos acercamos a la pausa descriptiva, pero combinados con elipsis audaces, que realizan saltos temporales como los

7 Así lo explicita en la entrevista realizada por Scott Tobias para The Dissolve, 18 de julio de 2014. 
referidos al cambio de año con una naturalidad que consigue dotar a Boyhood de ese ritmo tan acompasado con la vida que le otorga esa viva impresión de realidad. Cabría pensar aquí en nuevo eco baziniano, aventurar que el crítico francés también se rendiría ante Boyhood, como hizo ante Ladrón de bicicletas, para afirmar que "la desaparición de los actores, de la historia y de la puesta en escena desemboca finalmente en la perfecta ilusión estética de la realidad; en una más completa aparición del cine" (Bazin, 1990, p. 341).

Esa temporalidad longitudinal que configura la estructura narrativa de Boyhood y que le permite trabajar con mecanismos discursivos que potencian la hibridación entre ficción y documental se detiene, no obstante, ante una barrera clara, que la película de Linklater respeta sin ambages: la ausencia de autoconsciencia de los personajes, que los diferencia con nitidez de las personas que protagonizan los documentales longitudinales o los cineastas que han planteado sus filmografías en clave autobiográfica. El carácter serial y episódico de estos documentales se va construyendo sobre la creciente autoconsciencia de sus protagonistas, ya sean las personas ordinarias de las Up Series o los cineastas autobiográficos. De un modo u otro su discurso se articula sobre la experiencia pasada, subrayando, como ya mencionábamos, el carácter narrativo de su construcción identitaria, a través de esa incorporación autoconsciente del pasado en su presente existencial (algo que además los documentales longitudinales subrayan con el recurso a metraje de entregas anteriores). Los personajes de Boyhood, sin embargo, en cuanto caracteres de ficción, carecen de esa autoconsciencia que enfrenta a los protagonistas de los documentales con su periplo existencial real, privándoles de ese eco punzante que plantea el paso del tiempo en toda biografía personal. Además, tampoco Boyhood como relato otorga demasiado peso a la mirada retrospectiva de los personajes de ficción dentro de su universo diegético. Estamos más bien ante un relato en donde el tiempo presente tiene un protagonismo fundamental, en sintonía también con ese acercamiento más observacional del relato. Hay, sin embargo, una escena importante que se distancia de ese enfoque: se trata de la partida de 
Mason para la universidad. Tras un primer diálogo sobre objetos de la memoria -como aquella primera foto del hijo-, la madre hace patente la dimensión retrospectiva, cuando rompe a llorar ante su hijo:

You know what I'm realising? My life is just going to go. Like that. This series of milestones. Getting married. Having kids. Getting divorced. The time that we thought you were dyslexic. When I taught you how to ride a bike. Getting divorced... again. Getting my masters degree. Finally getting the job I wanted. Sending Samantha off to college. Sending you off to college. You know what's next? Huh? It's my fucking funeral!

Con todo, como se ha apuntado, se trata de una situación más bien excepcional en el conjunto del relato, cuyos personajes han estado planteados de modo habitual anclados en un presente con una fuerte proyección hacia el futuro, en buena medida también por la focalización del relato en los personajes más jóvenes.

\section{Conclusiones}

El balance de esta exploración del tiempo longitudinal en el cine nos muestra las poderosas virtualidades de este acercamiento para explorar las dinámicas humanas desplegadas en el tiempo. Su planteamiento narrativo consigue convocar el interés a través de relatos que suelen plantean miradas de corte más bien observacional, combinadas con un empleo atinado de las elipsis.

En el ámbito de la no-ficción, los documentales longitudinales combinan un retrato colectivo de una sociedad con una progresiva exploración de los protagonistas individuales, que acaba virando el enfoque de estas obras hacia una exploración de la identidad personal entendida como identidad narrativa. Esta tendencia se ve acentuada en las filmografías autobiográficas, en donde cada película va profundizando en esa exploración de la identidad personal, aunque siempre en diálogo con su contexto social, que ancla esas indagaciones en una escala de intereses más universal.

Estos asuntos tienen un reflejo muy sugerente en el cine de ficción en la propuesta cinematográfica que plantea Boyhood. A partir de un enfoque que 
busca el contagio creativo con recursos del cine de no-ficción, nos encontramos con un logrado retrato colectivo de la sociedad estadounidense, al tiempo que con un certero despliegue de las tramas de maduración de los personajes, articulado en una estructura narrativa en la que destaca el audaz y acertado uso de las elipsis.

\section{Referencias bibliográficas}

Adams, S. (2014). Interview: Richard Linklater Examines 'Boyhood,' Memory, Time \& $\quad$ Perspective. 17-7-2014. http://blogs.indiewire.com/theplaylist/interview-richard-linklaterexamines-boyhood-memory-time-perspective-20140715 Consultado 17-9-2015.

Bazin, A. (1990). Qué es el cine. Madrid: Rialp.

Cuevas, E. (2006). The Immigrant Experience in Jonas Mekas's Diary Films: A Chronotopic Análisis of Lost, Lost, Lost. Biography. 29/1, 55-73.

Cuevas, E. \& y Muguiro, C. (Eds.) (2002). El hombre sin la cámara. El cine de Alan Berliner / The Man without the Movie Camera: The Cinema of Alan Berliner. Madrid: Ediciones Internacionales Universitarias.

Cuevas, E. \& García, A. N. (Eds.) (2008). Landscapes of the Self: The Cinema of Ross McElwee / Paisajes del yo. El cine de Ross McElwee. Madrid: Ediciones Internacionales Universitarias.

Eakin, P. J. (2008). Living Autobiographically. How We Create Identity in Narrative. Ithaca, EEUU: Cornell University Press.

Genette, Gérard (1989). Figuras III. Barcelona: Lumen.

Highmore, B. (Ed.) (2002). Everyday Life Reader. Londres: Routledge.

Kilborn, R. (2010). Taking the Long View. A Study of Longitudinal Documentary. Manchester: Manchester University Press.

Kracauer S. (1989). Teoría del cine: la redención de la realidad física. Barcelona: Paidós.

Lejeune P. (1994). El pacto autobiográfico y otros estudios. Madrid: Megazul-Endymion.

Levi, G. (1993). Sobre microhistoria. En P. Burke (ed.), Formas de hacer historia (pp. 97-119).Madrid: Alianza.

Lüdtke, A. (Ed.) (1995). History of Everyday Life: Reconstructing Historical Experiences and Ways of Life. Ewing, EEUU: Princeton University Press.

Tobias, S. (2014). Richard Linklater discusses the 12-year journey to $\begin{array}{lllll}\text { Boyhood. } & \text { En } & \text { The } & \text { Dissolve. } & \text { 18-2014 }\end{array}$ 
https://thedissolve.com/features/interview/666-interview-richard-

linklater/ Consultado 20-9-2015.

Wickman, F. (2014). FAQ: How They Made Boyhood. En Slate Magazine. 117-2014

http://www.slate.com/blogs/browbeat/2014/o7/11/boyhood_movie explained_how_they_made_it_whose_boyhood_inspired_it_and_m ore.html. Consultado 18-9-2015.

\section{Películas citadas}

Apted, M. (1964-2012). Up Series. Gran Bretaña.

Arlyck, R. (2005). Following Sean. EEUU

Berliner, A. (1991). Intimate Stranger. EEUU

Berliner, A. (2012). First Cousin Once Removed. EEUU

De Sica, V. (1948). Ladri di biciclette. Italia

Hartleb, R. (1997-2006). The Children of Jordbrö. Suecia

Jordá, J. (1980). Numax presenta... España

Jordá, J. (2004). Veinte años después. España

Junge, W. (1961-2007). The Children of Golzow. Alemania

Linklater, R. (2014). Boyhood. EEUU

McElwee, R. (1984). Backyard. EEUU

McElwee, R. (1991). Something to Do with the Wall. EEUU

McElwee, R. (1993). Time Indefinite. EEUU

McElwee, R. (2011). Photographic Memory. EEUU

Mikhalkov, N. (1994). Anпа 6-18 (Анна: от 6 до 18). Rusia

Miroshnichenko, S. (1991-2012). Born in the USSR. Rusia

Ullrich, H. \& y Gumm, D. (1985-2004). Berlin-Ecke Bundesplatz. Alemania

Cómo citar: Cuevas Álvarez, E. (2016). "La temporalidad longitudinal en el cine: de las Up series a Boyhood". Fotocinema. Revista científica de cine y fotografía, $\quad \mathrm{n}^{\mathrm{O}} \quad 12, \quad \mathrm{pp}$ 221-236. Disponible: http://www.revistafotocinema.com/ 